\title{
Microscopic theory for radiation-induced zero-resistance states in 2D electron systems: Franck-Condon blockade
}

Jesús Iñarrea

Citation: Appl. Phys. Lett. 110, 143105 (2017); doi: 10.1063/1.4979830

View online: http://dx.doi.org/10.1063/1.4979830

View Table of Contents: http://aip.scitation.org/toc/apl/110/14

Published by the American Institute of Physics

\section{Articles you may be interested in}

Indium segregation in N-polar InGaN quantum wells evidenced by energy dispersive X-ray spectroscopy and atom probe tomography

Appl. Phys. Lett. 110, 143101143101 (2017); 10.1063/1.4979786

Photoresponse in gate-tunable atomically thin lateral MoS2 Schottky junction patterned by electron beam Appl. Phys. Lett. 110, 143109143109 (2017); 10.1063/1.4979831

Self-driven visible-blind photodetector based on ferroelectric perovskite oxides

Appl. Phys. Lett. 110, 142901142901 (2017); 10.1063/1.4979587

Single photon extraction from self-assembled quantum dots via stable fiber array coupling

Appl. Phys. Lett. 110, 142104142104 (2017); 10.1063/1.4979827

Optimal design of nanoporous materials for electrochemical devices

Appl. Phys. Lett. 110, 143103143103 (2017); 10.1063/1.4979466

$150 \mathrm{~mW}$ deep-ultraviolet light-emitting diodes with large-area AIN nanophotonic light-extraction structure emitting at $265 \mathrm{~nm}$

Appl. Phys. Lett. 110, 141106141106 (2017); 10.1063/1.4978855

\section{HDDEN}

\section{Instruments for Advanced Science}
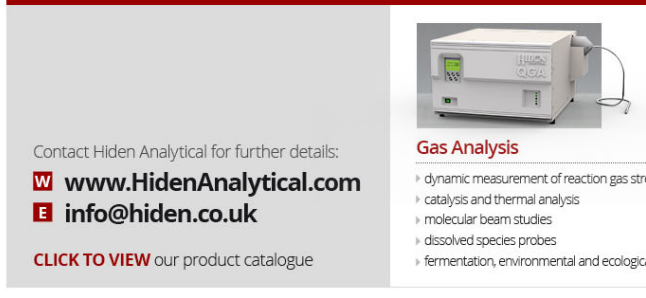

Gas Analysis

- dynamic measurement of reaction gas streams

cataysis and thermal analysis

molecuar beam sudes

dissolved species probes

fermentation, envirormental and ecological studies

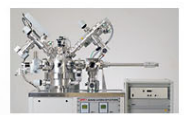

Surface Science

IUHVTPD
, SIMS

, end point detection in ion beam etch

, elemental imaging- sufface mapping

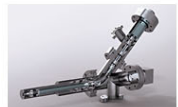

Plasma Diagnostics

, plasma source characterization etch and depositon process reaction kinetic studies

analysis of neutral and radical species

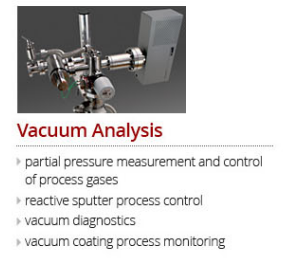




\title{
Microscopic theory for radiation-induced zero-resistance states in 2D electron systems: Franck-Condon blockade
}

\author{
Jesús Iñarrea \\ Escuela Politécnica Superior, Universidad Carlos III, Leganes, Madrid 28911, Spain and \\ Unidad Asociada al Instituto de Ciencia de Materiales, CSIC, Cantoblanco, Madrid 28049, Spain
}

(Received 6 February 2017; accepted 25 March 2017; published online 4 April 2017)

\begin{abstract}
We present a microscopic model on radiation-induced zero resistance states according to a novel approach: Franck-Condon physics and blockade. Zero resistance states rise up from radiation-induced magnetoresistance oscillations when the light intensity is strong enough. The theory begins with the radiation-driven electron orbit model that proposes an interplay of the swinging nature of the radiation-driven Landau states and the presence of charged impurity scattering. When the intensity of radiation is high enough, the driven-Landau states (vibrational states) involved in the scattering process are spatially far from each other and the corresponding electron wave functions no longer overlap. As a result, a drastic suppression of the scattering probability takes place and current and magnetoresistance exponentially drop. Finally, zero resistance states rise up. This is an application to magnetotransport in two-dimensional electron systems of the Franck-Condon blockade, based on the Franck-Condon physics which in turn stems from molecular vibrational spectroscopy. Published by AIP Publishing.
\end{abstract}

[http://dx.doi.org/10.1063/1.4979830]

Radiation-induced magnetoresistance $\left(R_{x x}\right)$ oscillations $(\mathrm{RIRO})^{1,2}$ turn up in high mobility two-dimensional electron systems (2DESs) under illumination at low temperature $(T \sim 1 K)$ and low magnetic fields $(B)$ perpendicular to the 2DES. When increasing radiation power $(P)$, maxima and minima oscillations increase, however the latter evolve into zero resistance states (ZRS). ${ }^{1,2}$ Many experiments ${ }^{3-22}$ and theoretical explanations ${ }^{23-39}$ have been proposed to understand these effects but no consensus among the people devoted to this field has yet been reached. Therefore, we have to admit that, to date, RIRO and ZRS are still open issues that remain in the cutting edge of condensed matter physics regarding the radiation-mater interaction. This is especially true in the case of ZRS, perhaps the most intriguing and challenging effect in this field. Despite the fact that plenty of theories have been developed for RIRO, when it comes to ZRS only a few theoretical models have been put forward. ${ }^{23,27,40,44,45}$ In general, they predict negative $R_{x x}$, while it was not experimentally confirmed. On the other hand, the most accepted theory on ZRS is based on the formation of current and electrical field domains; ${ }^{40}$ the key is the existence of an inhomogeneous current flowing through the sample due to the presence of a domain structure. Yet, this is a macroscopical model that overlooks any microscopic approach on ZRS.

In this letter, we develop a microscopic theory for ZRS that is based on the radiation-driven electron orbit model. This model, in turn, is based on the exact solution of the electronic wave function in the presence of a static magnetic field interacting with radiation and a perturbation treatment for elastic scattering due to randomly distributed charged impurities. This scattering between Landau states, LS (vibrational states), is successfully completed when there is a net overlap between the initial and final wave functions (see Fig. 1). In this model, the LS semiclassically describe orbits driven by radiation, "driven LS," whose center positions oscillate according to the radiation frequency. This radiation-driven oscillation alters dramatically the scattering conditions. In some cases, the LS advance during the scattering jump and, on average, the

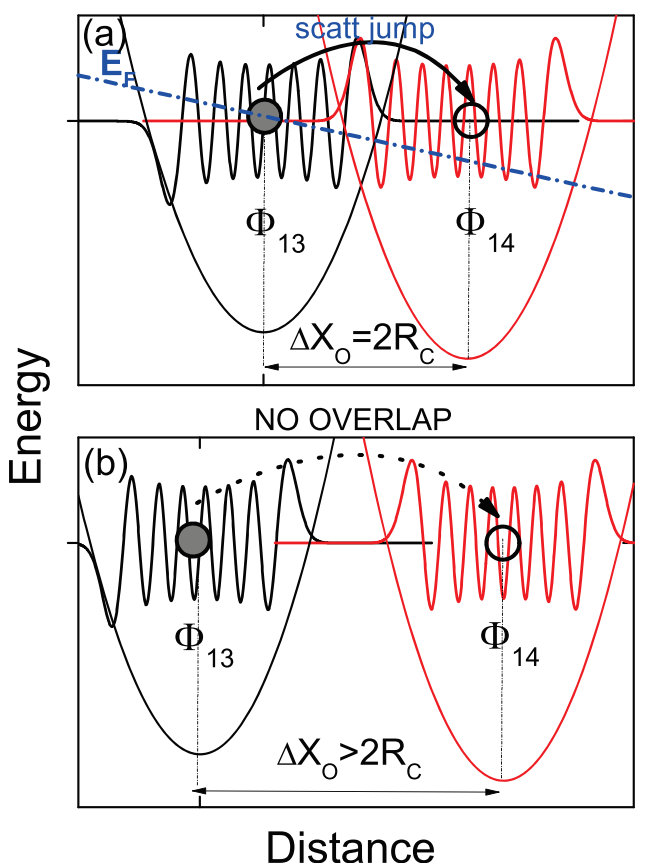

FIG. 1. Schematic diagrams of electron scattering between Landau states. In (a), there is an important overlap between Landau states. The case of $\Phi_{13}$ and $\Phi_{14}$ is shown as an example. Then, the charged impurity scattering is very likely to occur. For this to happen, it is essential that the distance between the guiding centers of the Landau states is around twice the cyclotron radius or less. In (b), we observe the opposite situation. Now the distance is bigger than twice the cyclotron radius and the overlap between the Landau states does not exist. Then, the scattering process is extremely unlikely to happen. The circles represent the guiding center of the Landau states. 
PEAK

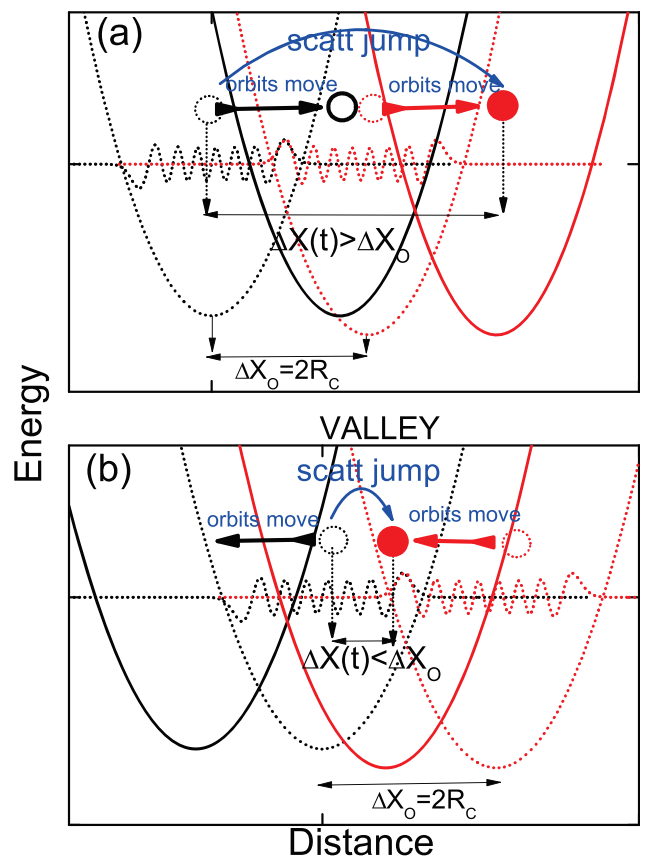

FIG. 2. Schematic diagram for scattering between Landau states in the presence of radiation. Under radiation, the Landau states are harmonically driven in a swinging motion with the radiation frequency. In (a), the Landau states move forward and on average the electrons advance further than the dark case (peaks). In (b), the Landau states move backward and on average the electrons advance less than in the dark case (valleys). For both panels, dotted parabolas represent the initial driven Landau states and the solid ones the final states after the scattering event. The circles represent the corresponding guiding center positions of the Landau states before (dotted) and after (solid) scattering.

advanced distance by electrons is bigger than in the dark giving rise to peaks in RIRO (see Fig. 2(a)). In others, the LS go backward during the jump and the net distance is smaller obtaining valleys (see Fig. 2(b)). But in all of them, there must be a net overlap of wave functions in order to have important and valuable contributions to $R_{x x}$.

This idea is similar to the one in Franck-Condon (FC) physics and extensively used in vibrational spectroscopy and molecular quantum mechanics. ${ }^{41,42}$ ZRS turn up when the radiation intensity is high enough. Then, it can happen that the final LS ends up behind the initial position of the scattering jump. Although this process corresponds to a good overlap between LS, the average advanced distance is equal to zero and does not contribute to $R_{x x}$. Then, we can consider other final LS much distant with respect to the scattering initial position. These LS could still end up ahead of that position during the scattering-driven process even at very high light intensities. Nevertheless, these LS do not significantly overlap and the corresponding contribution to $R_{x x}$ exponentially drops (see Fig. 3). As a result, scattering rate, current, and $R_{x x}$ are dramatically suppressed, electrons remain in their initial LS, and ZRS rise up. This effect is known as Franck-Condon blockade ${ }^{43}$ and it is at the heart of the physical origin of ZRS.

In the radiation-driven electron orbits model, the electron time-dependent Schrödinger equation with a timedependent force and magnetic field is exactly solved to study the magnetoresistance of a 2DES subjected to radiation at low $B$ and temperature, $T .^{23,46,47}$ Accordingly, the exact

\section{ZERO RESISTANCE STATES}

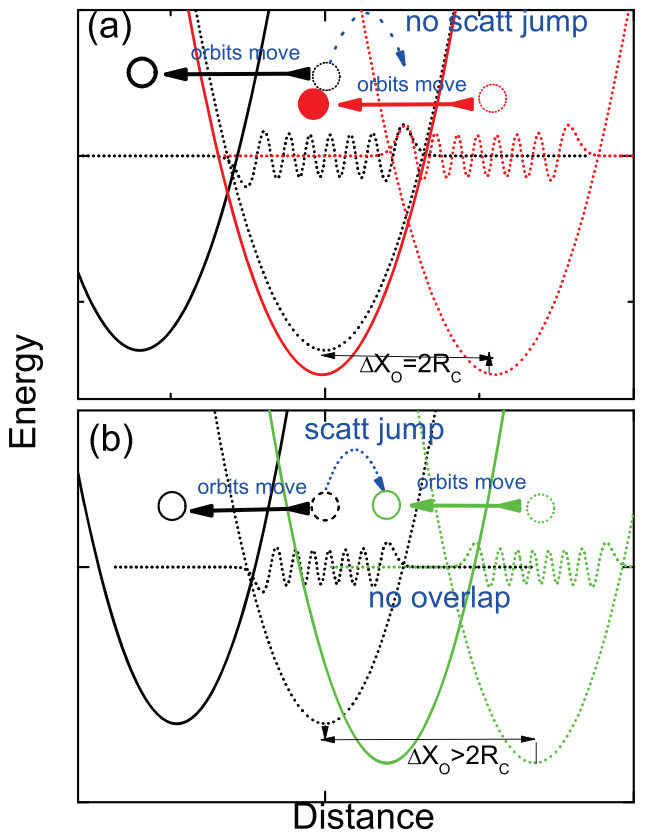

FIG. 3. Schematic diagram explaining the physical origin of ZRS. In (a), when the intensity of radiation is high enough and the Landau states move backwards it may happen that the final Landau state, initially at a distance of twice the cyclotron radius $\left(2 R_{c}\right)$, ends up behind the scattering initial position. Now and although the overlap is important, the average advanced distance is equal to zero. In (b), scattering processes to Landau states at more than $2 R_{c}$. These Landau states end up still ahead of the initial jump position and there is a positive advanced distance. However, the overlap between these involved Landau states is negligible and the final magnetoresistance exponentially drops and ZRS show up.

expression of the obtained electronic wave function reads $\Psi_{n}(x, t) \propto \phi_{n}\left(x-X_{0}-x_{c l}(t), t\right)$, where $\phi_{n}$ is the solution for the Schrödinger equation of the unforced quantum harmonic oscillator. Thus, the obtained wave function (Landau state or Landau orbit) is the same as the one of the standard quantum harmonic oscillators where the guiding center, $X_{0}$, without radiation, is displaced by $x_{c l}(t) . x_{c l}(t)$ is the classical solution of a negatively charged, forced and damped, harmonic oscillator

$$
\begin{aligned}
x_{c l}(t) & =\frac{-e E_{o}}{m^{*} \sqrt{\left(w_{c}^{2}-w^{2}\right)^{2}+\gamma^{4}}} \cos (w t-\beta) \\
& =-A \cos (w t-\beta),
\end{aligned}
$$

where $E_{0}$ is the intensity of radiation, $w$ is the radiation frequency, and $w_{c}$ is the cyclotron frequency. $\gamma$ is a phenomenologically introduced damping factor for the electronic interaction with acoustic phonons. $\beta$ is the phase difference between the radiation-driven guiding center and the driving radiation itself and it is given by $\tan \beta=\frac{\gamma^{2}}{w_{c}^{2}-w^{2}}$. Thus, the guiding center lags behind radiation a phase constant of $\beta$. When the damping parameter $\gamma$ is important $\left(\gamma>w \Rightarrow \gamma^{2} \gg w^{2}\right)$, then $\tan \beta \rightarrow \infty$ and $\beta \rightarrow \frac{\pi}{2}$. Now, the time-dependent guiding center is $X=X_{0}+x_{c l}=X_{0}-A \sin w t$. This physically implies that the orbit guiding centers oscillate harmonically at the radiation frequency $w$, but radiation leads the guiding center displacement in $\frac{\pi}{2}$. 
The longitudinal conductivity $\sigma_{x x}$ in the 2DES is obtained applying the Boltzmann transport theory. With this theory and within the relaxation time approximation, $\sigma_{x x}$ is given by the following equation: ${ }^{48-54}$

$$
\sigma_{x x}=e^{2} \int_{0}^{\infty} d E \rho_{i}(E)(\Delta X)^{2} W_{I}\left(-\frac{d f(E)}{d E}\right),
$$

with $E$ being the energy and $\rho_{i}(E)$ the density of initial Landau states. $W_{I}$ is the remote charged impurity scattering rate, given, according to the Fermi's Golden Rule, by $W_{I}$ $=N_{I} \frac{2 \pi}{\hbar}\left|\left\langle\phi_{m}\left|V_{s}\right| \phi_{n}\right\rangle\right|^{2} \delta\left(E_{m}-E_{n}\right)$, where $N_{I}$ is the impurity density and $E_{n}$ and $E_{m}$ are the energies of the initial and final LS, respectively. $V_{s}$ is the scattering potential for charged impurities, ${ }^{53} \Delta X$ is the average distance advanced by the electron between orbits in every scattering jump in the $x$ direction and is given by, ${ }^{55} \Delta X=\Delta X^{0}-A \sin \left(2 \pi \frac{w}{w_{c}}\right) \cdot \Delta X^{0}$ is the distance between the guiding centers of the LS involved in the scattering event. Since all LS oscillate in phase, this distance remains constant during the driving motion and is the same with or without radiation.

After some algebra, we get to an expression for $\sigma_{x x}^{56,57}$

$$
\begin{aligned}
\sigma_{x x}= & \frac{e^{2} m^{*}}{\pi \hbar^{2}}\left[\Delta X^{0}-A \sin \left(2 \pi \frac{w}{w_{c}}\right)\right]^{2} \\
& \times W_{I}\left[1+\frac{2 X_{s}}{\sinh \left(X_{s}\right)} e^{-\frac{\pi \Gamma}{h w_{c}}} \cos \left(\frac{2 \pi E_{F}}{\hbar w_{c}}\right)\right],
\end{aligned}
$$

where $X_{s}=\frac{2 \pi^{2} k_{B} T}{\hbar w_{c}}, k_{B}$ is the Boltzmann constant, $\Gamma$ is the Landau level width, and $E_{F}$ is the Fermi energy. To find the expression of $R_{x x}$, we use the well-known tensorial relation $R_{x x}=\frac{\sigma_{x x}}{\sigma_{x x}^{2}+\sigma_{x y}^{2}} \simeq \frac{\sigma_{x x}}{\sigma_{x y}^{2}}$, where $\sigma_{x y} \simeq \frac{n_{e} e}{B}, n_{e}$ is the electron density, and $\sigma_{x x} \ll \sigma_{x y}$.

To apply the Franck-Condon physics to the problem of ZRS, we need to properly develop the matrix element inside the scattering rate $W_{I}$. This matrix element can be expressed as $^{52-54}$

$$
\left|\left\langle\phi_{m}\left|V_{s}\right| \phi_{n}\right\rangle\right|^{2}=\sum_{q}\left|V_{q}\right|^{2}\left|I_{n m}\right|^{2} \delta_{k_{y}^{\prime}, k_{y}+q_{y}},
$$

where $V_{q}=\frac{e^{2}}{\epsilon\left(q+q_{s}\right)}, \epsilon$ is the dielectric constant, and $q_{s}$ is the Thomas-Fermi screening constant. ${ }^{53}$ The integral $I_{n m}$ is given by

$$
I_{n m}=\int_{-\infty}^{\infty} e^{i q_{x} x} \phi_{m}\left(x-X^{\prime}\right) \phi_{n}(x-X) d x,
$$

where $X=X_{0}-A \sin w t$ and $X^{\prime}=X_{0}^{\prime}-A \sin w t$ are the guiding centers of $\phi_{n}$ and $\phi_{m}$, respectively. Expanding the exponential in the integral in powers of $q_{x} x: e^{i q_{x} x}=1+i q_{x} x$ $-\frac{1}{2} q_{x}^{2} x^{2}-\cdots$. On the one hand and using a screened Coulomb potential, $\mathrm{x}$ is of the order of the Thomas-Fermi screening length $1 / q_{s}, x \sim 1 / q_{s} \simeq 5 \times 10^{-9} \mathrm{~m}$ for GaAs. ${ }^{58}$ On the other hand, $q_{x} \sim q=2 k_{F} \sin \frac{\theta}{2}$ (Ref. 58) where $\theta$ is the scattering angle and $k_{F}$ is the Fermi wave vector. For high mobility samples, the scattering is mainly described by long range, small angle (charged impurity) scattering. Then, we assume that for the samples used in experiments this angle is small or very small. ${ }^{59}$ We have taken an average scattering angle of $\theta \leq 10^{\circ}$ and for the Fermi wave vector $2 k_{F} \simeq(3-1) \times 10^{8} \mathrm{~m}^{-1}$ for a $2 \mathrm{DES}$ with the experimental electron density. ${ }^{1}$ This gives for $q_{x} \sim 10^{6}-10^{7} \mathrm{~m}^{-1}$ and then $q_{x} x \sim 10^{-3}-10^{-2} \ll 1$. We therefore make a good approximation retaining only the first term in the above expansion: $e^{i q_{x} x} \rightarrow 1$. The final outcome is that the integral $I_{n m}$ becomes an overlap integral of the LS involved in the scattering process: $I_{n m}=\int_{-\infty}^{\infty} \phi_{m}\left(x-X^{\prime}\right) \phi_{n}(x-X) d x$. This result implies that an important overlap between the initial and final LS will give, through the term $\left|I_{n m}\right|^{2}$, an intense scattering and in turn an intense $R_{x x}$. This principle is known in FranckCondon physics and extensively used in molecular vibrational spectroscopy. ${ }^{41,42}$ We translate it now into magnetotransport in the 2DES and calculate the square of the vibrational overlap integral, $\left|I_{n m}\right|^{2}$, the Franck-Condon factor. The expression for the Franck-Condon factor (FC) reads ${ }^{60}$

$$
\left|I_{n m}\right|^{2}=\frac{n !}{m !}\left[\frac{\Delta X_{0}^{2}}{2 R^{2}}\right]^{m-n} e^{-\frac{\Delta X_{0}^{2}}{2 R^{2}}}\left[L_{n}^{m-n}\left(\frac{\Delta X_{0}^{2}}{2 R^{2}}\right)\right]^{2},
$$

where $m \geq n, R^{2}=\frac{\hbar}{e B}$ is the square of the magnetic length and $L_{n}^{m-n}$ is the associate Laguerre polynomials.

In Fig. 4, we show the calculated FC factor versus $\Delta X_{0}$ in units of cyclotron radius $\left(R_{c}\right)$ for three different $B$ : $B=0.1,0.2$ and $0.3 \mathrm{~T}$. For each case, we also present the Landau level index for the Fermi energy and the scattering process considered in the simulation. We observe, as expected, that the FC factor presents important values only when $\Delta X_{0} \leq 2 R_{c}$ (important overlap between LS) and exponentially drops when $\Delta X_{0}>2 R_{c}$ (negligible overlap). As in vibrational transitions in infrared molecular spectroscopy

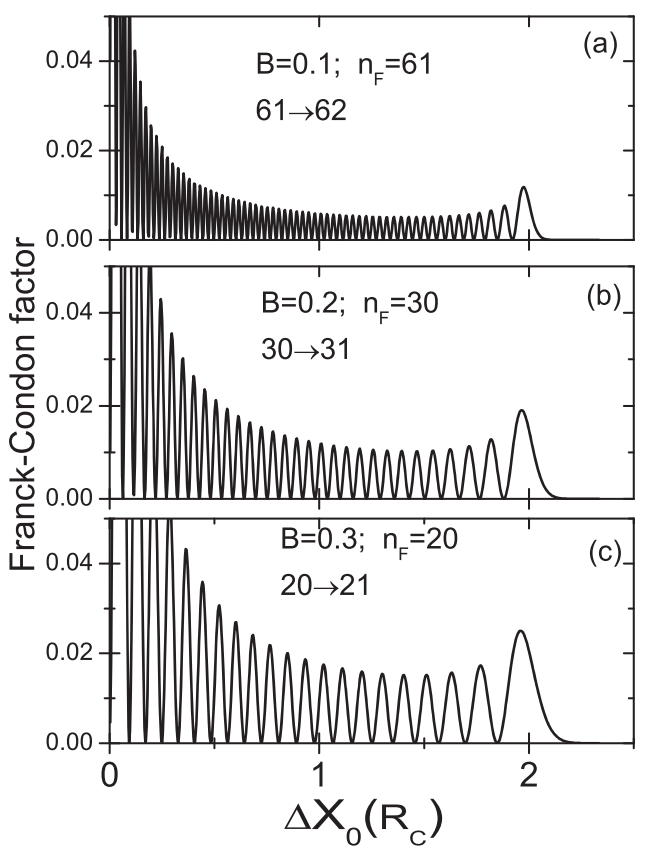

FIG. 4. Franck-Condon factor vs $\Delta X_{0}$ in units of cyclotron radius $R_{c}$ for three different $B: B=0.1,0.2$, and $0.3 \mathrm{~T}$. In each panel, we present also the Landau level index for the Fermi energy and the Landau levels indexes for the scattering process considered in the simulation. 


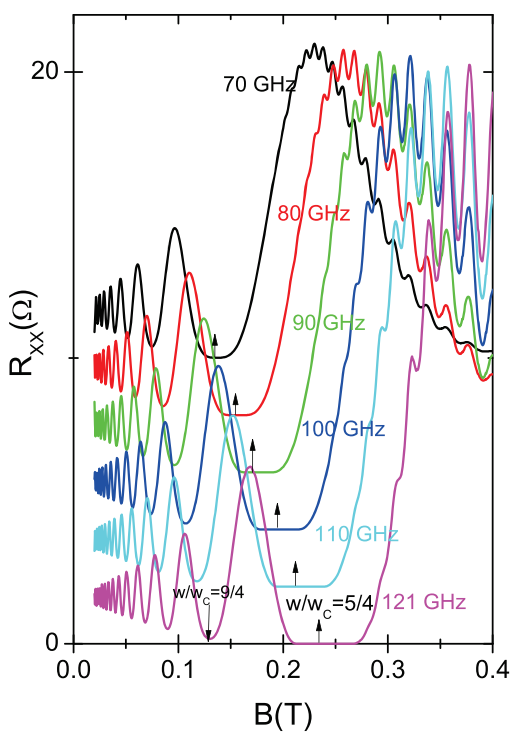

FIG. 5. Calculated $R_{x x}$ vs $B$ under illumination for different radiation frequencies to study the dependence of radiation-induced oscillations on the frequency.

with the spectroscopic lines, here the FC factor defines the intensity of the scattering. Thus, when the LS involved in the scattering event are at a distance of $2 R_{c}$ or less than the FC factors (see Fig. 4), $W_{I}$ gives important and nonnegligible values. Now, with a not very intense radiation, the final driven LS always ends up ahead of the LS initial position of scattering giving rise to bigger or smaller $\Delta X$ : peaks or valleys, respectively, in $R_{x x}$. This is described in Fig. 2. We can get to a totally different scenario if we further increase $P$ reaching a situation where the final LS ends up behind the initial scattering jump position and then, although with an important value for the FC factor, the average advance distance is zero. Nevertheless, we can consider further away LS at more distance than $2 R_{c}$ so that they end up, even at high $P$, ahead of the initial scattering position giving a net advanced distance. Yet, there is no overlap now and the FC factor turns out to be negligible. This physical scenario corresponds to the rise up of ZRS. This situation is described in Fig. 3. Figure 5 shows calculated $R_{x x}$ vs $B$ for different radiation frequencies. ZRS positions move according to the change of radiation frequency $w$, keeping the ratio, $\frac{w}{w_{c}}=j+\frac{1}{4} \cdot{ }^{37}$ Simulated ZRS are very clearly obtained for $j=1$.

This work was supported by the MINECO (Spain) under Grant No. MAT2014-58241-P and ITN Grant No. 234970 (EU). GRUPO DE MATEMATICAS APLICADAS A LA MATERIA CONDENSADA, (UC3M), Unidad Asociada al CSIC.

${ }^{1}$ R. G. Mani, J. H. Smet, K. von Klitzing, V. Narayanamurti, W. B. Johnson, and V. Umansky, Nature 420, 646 (2002).

${ }^{2}$ M. A. Zudov, R. R. Lu, N. Pfeiffer, and K. W. West, Phys. Rev. Lett. 90, 046807 (2003).

${ }^{3}$ A. N. Ramanayaka, R. G. Mani, J. Inarrea, and W. Wegscheider, Phys. Rev. B 85, 205315 (2012).

${ }^{4}$ R. G. Mani, V. Narayanamurti, K. von Klitzing, J. H. Smet, W. B. Johnson, and V. Umansky, Phys. Rev. B 69, 161306(R) (2004).

${ }^{5}$ R. G. Mani, J. H. Smet, K. von Klitzing, V. Narayanamurti, W. B. Johnson, and V. Umansky, Phys. Rev. Lett. 92, 146801 (2004).
${ }^{6}$ R. G. Mani, J. H. Smet, K. von Klitzing, V. Narayanamurti, W. B. Johnson, and V. Umansky, Phys. Rev. B 69, 193304 (2004).

${ }^{7}$ R. G. Mani, Physica E 22, 1 (2004).

${ }^{8}$ J. H. Smet, B. Gorshunov, C. Jiang, L. Pfeiffer, K. West, V. Umansky, M. Dressel, R. Meisels, F. Kuchar, and K. von Klitzing, Phys. Rev. Lett. 95, 116804 (2005).

${ }^{9}$ Z. Q. Yuan, C. L. Yang, R. R. Du, L. N. Pfeiffer, and K. W. West, Phys. Rev. B 74, 075313 (2006).

${ }^{10}$ T. Ye, H.-C. Liu, Z. Wang, W. Wegscheider, and R. G. Mani, Sci. Rep. 5, 14880 (2015).

${ }^{11}$ R. G. Mani and A. Kriisa, Sci. Rep. 3, 3478 (2013).

${ }^{12}$ R. G. Mani, Appl. Phys. Lett. 85, 4962 (2004).

${ }^{13}$ R. G. Mani, W. B. Johnson, V. Umansky, V. Narayanamurti, and K. Ploog, Phys. Rev. B 79, 205320 (2009).

${ }^{14}$ S. Wiedmann, G. M. Gusev, O. E. Raichev, A. K. Bakarov, and J. C. Portal, Phys. Rev. Lett. 105, 026804 (2010).

${ }^{15}$ S. Wiedmann, G. M. Gusev, O. E. Raichev, A. K. Bakarov, and J. C. Portal, Phys. Rev. B 81, 085311 (2010).

${ }^{16}$ D. Konstantinov and K. Kono, Phys. Rev. Lett. 103, 266808 (2009).

${ }^{17}$ S. I. Dorozhkin, L. Pfeiffer, K. West, K. von Klitzing, and J. H. Smet, Nat. Phys. 7, 336-341 (2011).

${ }^{18}$ R. G. Mani, C. Gerl, S. Schmult, W. Wegscheider, and V. Umansky, Phys. Rev. B 81, 125320 (2010).

${ }^{19}$ R. G. Mani, A. N. Ramanayaka, and W. Wegscheider, Phys. Rev. B 84, 085308 (2011).

${ }^{20}$ J. Inarrea, R. G. Mani, and W. Wegscheider, Phys. Rev. 82, 205321 (2010).

${ }^{21}$ T. Herrmann, I. A. Dmitriev, D. A. Kozlov, M. Schneider, B. Jentzsch, Z. D. Kvon, P. Olbrich, V. V. Belkov, A. Bayer, C. Schuh, D. Bougeard, T. Kuczmik, M. Oltscher, D. Weiss, and S. D. Ganichev, Phys. Rev. B 94, 081301 (2016).

${ }^{22}$ T. Ye, J. Iñarrea, W. Wegscheider, and R. G. Mani, Phys. Rev. B 94, 035305 (2016).

${ }^{23}$ J. Iñarrea and G. Platero, Phys. Rev. Lett. 94, 016806 (2005).

${ }^{24}$ A. C. Durst, S. Sachdev, N. Read, and S. M. Girvin, Phys. Rev. Lett. 91, 086803 (2003).

${ }^{25}$ X. L. Lei and S. Y. Liu, Phys. Rev. Lett. 91, 226805 (2003).

${ }^{26}$ V. I. Ryzhii, R. A. Suris, and B. S. Shchamkhalova, Sov. Phys. Semicond. 20, 1299 (1986).

${ }^{27}$ P. H. Rivera and P. A. Schulz, Phys. Rev. B 70, 075314 (2004).

${ }^{28}$ M. G. Vavilov et al., Phys. Rev. B 70, 161306 (2004).

${ }^{29}$ J. Inarrea and G. Platero, Nanotechnology 21, 315401 (2010).

${ }^{30}$ J. Iñarrea, Appl. Phys. Lett. 90, 262101 (2007).

${ }^{31}$ J. Inarrea, G. Platero, and A. H. MacDonald, Phys. Status Solidi A 203, 1148 (2006).

${ }^{32}$ J. Iñarrea, Appl. Phys Lett. 100, 242103 (2012).

${ }^{33}$ V. Ryzhii and R. Suris, J. Phys.:Condens. Matter 15, 6855 (2003).

${ }^{34}$ V. Ryzhii, Phys. Rev. B 68, 193402 (2003).

${ }^{35}$ V. Ryzhii and V. Vyurkov, Phys. Rev. B 68, 165406 (2003).

${ }^{36}$ J. Shi and X. C. Xie, Phys. Rev. Lett. 91, 086801 (2003).

${ }^{37}$ J. Iñarrea, Europhys. Lett. 113(5), 57004 (2016).

${ }^{38}$ Y. M. Beltukov and M. I. Dyakonov, Phys. Rev. Lett. 116, 176801 (2016).

${ }^{39}$ A. V. Andreev, I. L. Aleiner, and A. J. Millis, Phys. Rev. Lett. 91, 056803 (2003).

${ }^{40}$ F. S. Bergeret, B. Huckestein, and A. F. Volkov, Phys. Rev. B 67, 241303 (2003).

${ }^{41}$ I. N. Levine, Molecular Spectroscopy (John Wiley and Sons, New York, 1975).

${ }^{42} \mathrm{P}$. Atkins and R. Friedman, Molecular Quantum Mechanics (Oxford University Press, New York, 2005).

${ }^{43}$ J. Koch, F. von Oppen, and A. V. Andreev, Phys. Rev. B 74, 205438 (2006).

${ }^{44}$ S. A. Mikhailov, Phys. Rev. B 89, 045410 (2014).

${ }^{45}$ O. V. Zhirov, A. D. Chepelianskii, and D. L. Shepelyansky, Phys. Rev. B 88, 035410 (2013).

${ }^{46}$ E. H. Kerner, Can. J. Phys. 36, 371 (1958).

${ }^{47}$ K. Park, Phys. Rev. B 69, 201301(R) (2004).

${ }^{48}$ S. Titeica, Ann. Phys. 22, 128 (1935).

${ }^{49}$ V. Ryzhii, Sov. Phys. Solid State 11, 2078 (1970).

${ }^{50}$ A. D. Malov and V. I. Ryzhií, Sov. Phys. Solid State 14, 1766 (1973).

${ }^{51}$ V. I. Vyurkov, A. D. Gladun, A. D. Malov, and V. I. Ryzhií, Sov. Phys. Solid State 19, 2113 (1977).

${ }^{52}$ B. K. Ridley, Quantum Processes in Semiconductors, 4th ed. (Oxford University Press, 1993).

${ }^{53}$ T. Ando, A. Fowler, and F. Stern, Rev. Mod. Phys. 54, 437 (1982).

${ }^{54}$ B. M. Askerov, Electron Transport Phenomena in Semiconductors (World Scientific, Singapore, 1994). 
${ }^{55}$ J. Inarrea and G. Platero, Phys. Rev. B 84, 075313 (2011).

${ }^{56}$ N. Miura, Physics of Semiconductors in High Magnetic Fields (Oxford University Press, 2008).

${ }^{57}$ T. Ihn, Semiconductor Nanostructures. Quantum States and Electronic Transport (Oxford University Press, New York, 2010).

${ }^{58}$ J. H. Davies, The Physics of Low-dimensional Semiconductors (Cambridge University Press, 1998).
${ }^{59}$ G. Marchetti, M. Hodgson, J. McHugh, R. Chantrell, and I. D'Amico, Materials 7, 2795 (2014).

${ }^{60}$ R. G. Compton, Electron Tunneling in Chemistry (Elsevier, Amsterdam, 1989); W. Barford, Electronic and Optical Properties of Conjugated Polymers (Oxford Science Publications/Clarendon Press, Oxford, 2005); E. D. Zhebrak, preprint arXiv:150.06052v1 (2015); S.-H. Dong, Factorization Method in Quantum Mechanics (Springer, 2007). 